\title{
National comparisons of lung cancer survival in England, Norway and Sweden 2001-2004: differences occur early in follow-up
}

\author{
Lars Holmberg, ${ }^{1}$ Fredrik Sandin, ${ }^{2}$ Freddie Bray, ${ }^{4}$ Mike Richards, ${ }^{5}$ James Spicer, ${ }^{1}$ \\ Mats Lambe, ${ }^{3}$ Åsa Klint, ${ }^{6}$ Mick Peake, ${ }^{7}$ Trond-Eirik Strand, ${ }^{4}$ Karen Linklater, ${ }^{1}$ \\ David Robinson, ${ }^{1}$ Henrik Møller ${ }^{1}$
}

${ }^{1}$ Division of Cancer Studies, King's College London, School of Medicine, London, UK

${ }^{2}$ Regional Oncological Centre, Uppsala/Örebro Region,

Uppsala, Sweden

${ }^{3}$ Department of Medical

Epidemiology and Biostatistics,

Karolinska Institutet, Stockholm, Sweden

${ }^{4}$ Norwegian Cancer Registry, Oslo, Norway

${ }^{5}$ Department of Palliative Medicine, St Thomas' Hospital, London, UK

${ }^{6}$ Swedish Cancer Registry, National Board of Health and Welfare, Stockholm, Sweden 'Department of Respiratory Medicine, Glenfield Hospital, Leicester, UK

\section{Correspondence to}

Professor Lars Holmberg, Division of Cancer Studies, King's College London, School of Medicine, Research Oncology, 3rd Floor Bermondsey Wing, Guy's Hospital, London SE1 9RT, UK; lars.holmberg@kcl.ac.uk

Received 24 July 2009 Accepted 16 February 2010

\section{ABSTRACT}

Background Countries with a similar expenditure on healthcare within Europe exhibit differences in lung cancer survival. Survival in lung cancer was studied in 2001-2004 in England, Norway and Sweden.

Methods Nationwide cancer registries in England, Norway and Sweden were used to identify 250828 patients with lung cancer from England, 18386 from Norway and 24886 from Sweden diagnosed between 1996 and 2004, after exclusion of patients registered through death certificate only or with missing, zero or negative survival times. 5-Year relative survival was calculated by application of the period approach. The excess mortality between the countries was compared using a Poisson regression model.

Results In all subcategories of age, sex and follow-up period, the 5-year survival was lower in England than in Norway and Sweden. The age-standardised survival estimates were $6.5 \%, 9.3 \%$ and $11.3 \%$ for men and $8.4 \%, 13.5 \%$ and $15.9 \%$ for women in the respective countries in 2001-2004. The difference in excess risk of dying between the countries was predominantly confined to the first year of follow-up. The relative excess risk ratio during the first 3 months of follow-up comparing England with Norway 2001-2004 varied between 1.23 and 1.46, depending on sex and age, and between 1.56 and 1.91 comparing England with Sweden.

Conclusion Access to healthcare and population awareness are likely to be major reasons for the differences, but it cannot be excluded that diagnostic and therapeutic activity play a role. Future improvements in lung cancer management may be seen early in follow-up.

\section{INTRODUCTION}

Despite increasing overall cancer survival and diminishing regional differences within Europe, there are still differences between countries with similar national healthcare systems and total national expenditure on healthcare. ${ }^{1}{ }^{2}$ Survival differences are generally most pronounced for cancers with good survival, indicating that these differences may be sensitive to the allocation of health resources. Differences are less pronounced for those cancers with a good prognosis which are treated at specialised centres (eg, testicular cancer) and for cancers with a poor prognosis (eg, lung cancer). ${ }^{13}$ An understanding of the differences in survival on a population level, even for cancers with a poor prognosis, may reveal critical points in the infrastructure of healthcare that are important areas for improvement.

Unless detected at a localised and resectable stage, lung cancer is a rapidly fatal disease and the effective therapeutic arsenal is limited. EUROCARE 4 reported a 5-year relative survival for the followup period $2000-2002$ of $8.4 \%$ in the UK, $11.2 \%$ in Norway and 13.9\% in Sweden. ${ }^{2}$ Between 1990 and 1994, and 2000 and 2002 there was a relative improvement in survival of $20-35 \%$ in Norway and Sweden, but $<10 \%$ in the UK. ${ }^{2}$

We studied patterns of relative survival during 2001-2004 in patients with lung cancer diagnosed in 1996-2004 in England, Norway and Sweden. These three countries have a similar expenditure on healthcare, and all have population-based cancer registration systems. We were particularly interested in identifying the periods of follow-up where the major differences in survival between the countries occur, and in studying the impact on survival of age at diagnosis.

\section{METHODS}

\section{Cancer registration in England}

Data are collected by eight regional cancer registries. ${ }^{4}{ }^{5}$ The means of collecting the data vary between registries, from largely automated systems based on electronic pathology and hospital activity records, to manual operations where extraction of information from clinical records plays a large part. Electronic data sources are being increasingly used. The regional data are collated into a national data repository maintained by the Office for National Statistics and the National Cancer Intelligence Network. ${ }^{6}$ These processes resolve cross-registry duplicates and provide continuous update of the vital status and cause of death for each person. Data linkage is achieved via the National Health Service (NHS) number, which is a unique personlevel identifier for all English citizens registered with a general practitioner in the NHS.

\section{Cancer registration in Norway}

The Cancer Registry of Norway collects notifications on cancer in the Norwegian population. Hospitals, pathological laboratories, general practitioners and the National Statistics Office (Statistics Norway) are the primary sources, with clinical notifications reported on structured templates and pathology notifications routinely sent to the Registry. Statistics Norway provides information 
on the cause of death noted on death certificates, and updates the vital status of all registered persons on a monthly basis. Since 1998 the Registry has received discharge diagnoses for all patients treated for malignant and premalignant conditions in every hospital and outpatient clinic, and these are used as a basis for sending out reminders. Patients are identified through a unique 11-digit personal identification number assigned to all people residing in Norway. Through record linkage, this provides a reliable means of tracking patients and avoiding duplicate registrations. A recent review of data quality showed an estimated completeness of lung cancer registration of $96.9 \%$ for 2001-2005.

\section{Cancer registration in Sweden}

The Swedish Cancer Registry covers the entire Swedish population. It is compulsory for every healthcare provider to report newly detected cancers at clinical, morphological or other laboratory examination, and for those diagnosed at autopsy. The Registry receives one report from the laboratory examinations and one from the clinical department. The primary data are collected at a Regional Cancer Registry, which is responsible for coding, verification and correction. Cases without cancer notification according to the above requirements, but reported to the Cause of Death Registry (cases denoted as DCO, death certificate only, and DCN, death certificate notification), are not included in the Swedish Cancer Registry. Patients are identified through an individually unique identification number assigned to all people residing in Sweden, allowing for record linkage, and means of tracking patients and avoiding duplicates. Cancer registration was validated for the year 1998 against the Swedish Hospital Discharge Registry and indicated a 6\% under-reporting of lung cancer, more pronounced in elderly patients without a histopathological diagnosis and not actively treated. ${ }^{8}$

\section{Statistical methods}

Patients with lung cancer diagnosed between 1996 and 2004 were identified from the national registries of the three countries. We excluded all DCO cases from Norway and England, and patients with either a missing or zero survival time, or a negative survival time as a result of erroneous reporting dates. In the Norwegian data there was evidence of a preference for day 1 of the month to be set as the day of diagnosis: $\sim 15 \%$ of cases contributing follow-up in the period 2001-2004 were registered to the first day of the month. To reduce bias due to this effect, the day of diagnosis was changed from day 1 to day 15 of the month for Norwegian patients diagnosed in years where the proportion of patients originally recorded as being diagnosed on day 1 was more than twice the expected proportion (1996-2003). We then assigned a survival of 1 day to those cases that acquired a zero or negative survival time as a result of this procedure. There was no such digit preference in the English or Swedish data, and original dates were used.

5-Year relative survival was calculated as the ratio of the observed survival in the study population to the expected survival of the background population, ${ }^{9}$ the latter derived from life tables of age-and sex-specific death rates for England, Norway and Sweden. Up to 5 years of follow-up during 2001-2004 were analysed by application of the period approach suggested by Brenner and Gefeller. ${ }^{10}$ Using this approach, the estimates are based on the survival times of patients diagnosed in 1996-2004, although only the person-time at risk and events (death or censoring) occurring during 2001-2004 are taken into account. Four different follow-up intervals were considered: 0-3 months, 3 months -1 year, $1-2$ years and $2-5$ years.
Plots of the excess number of deaths per 100 person-yearsthat is, the difference between the observed number of deaths and the expected number of deaths, by country, sex, age group and follow-up interval-were produced. Thereafter, the excess mortality rate was modelled separately for men and women, using the Poisson regression model of Dickman et al, ${ }^{11}$ with follow-up interval, age at diagnosis and country as explanatory factors. In order to assess the relative excess mortality between countries across follow-up intervals and age groups, interaction terms were included in the model. The model fit was verified by residual analysis, and by evaluating the deviance of the model and the corresponding degrees of freedom, against the $\chi^{2}$ distribution on the same number of degrees of freedom.

Relative survival analysis was performed with the statistical software package Stata ${ }^{12}$ using the procedure strs developed by Paul Dickman. Further analyses were performed using the $\mathrm{R}$ statistical software package. ${ }^{13}$

\section{RESULTS \\ Descriptive statistics}

We identified 282142 patients from England, 19013 from Norway and 26722 from Sweden diagnosed with lung cancer between 1996 and 2004. The proportion of patients with DCOs in England (6.8\%) was greater than in Norway (1.0\%), whereas in Sweden this source is not used. In England, Norway and Sweden there were $4.3 \%, 2.3 \%$ and $6.9 \%$ of patients, respectively, with a missing, negative or zero survival time. After exclusion of these cases, 250828 patients from England, 18386 from Norway and 24886 from Sweden were available for analysis (figure 1). In all three countries there were more men than women with lung cancer, the proportion of men varying between $57.8 \%$ in Sweden and $63.3 \%$ in Norway. A larger proportion of patients were over 70 in England $(58.2 \%)$ as compared with Norway (50.3\%) and Sweden (47.9\%). In all countries together, $\sim 65 \%$ of patients were aged $60-79$ (table 1 ).

\section{5-Year cumulative relative survival}

In all subcategories of age and sex, 5-year cumulative survival was lower in England than in Norway and Sweden (table 2). 5 Year cumulative survival was broadly similar in Norway and Sweden in the younger age groups, but for patients over 60 in Norway survival was intermediate between that in England and Sweden. The absolute differences in 5-year survival between Sweden and England were similar over age groups. Women had a better survival than men in all strata of age and country, and the sex-specific differences were greater (in absolute terms) in younger than in older patients. In the sex-specific comparisons between the countries, the absolute differences were larger among women than among men (table 2).

\section{Excess deaths per 100 person-years by age group and follow-up interval}

When the comparisons between the countries were stratified by follow-up interval and age group, the absolute rates of and the differences in the excess risk of dying were much greater during the first year-and most markedly the first 3 months-of follow-up. The differences between the countries were evident across all age groups, with the exception that Norway and Sweden converged among younger age groups (figure 2). Separate analyses by sex gave results very similar to the analysis of all patients combined (data not shown). Table 3 illustrates the same comparison in relative terms, showing the ratios of excess risk of death in the different countries. The differences were not confined to any specific age group and were most marked during 
Figure 1 Flowchart of inclusion/ exclusion in the data sets for analysis.

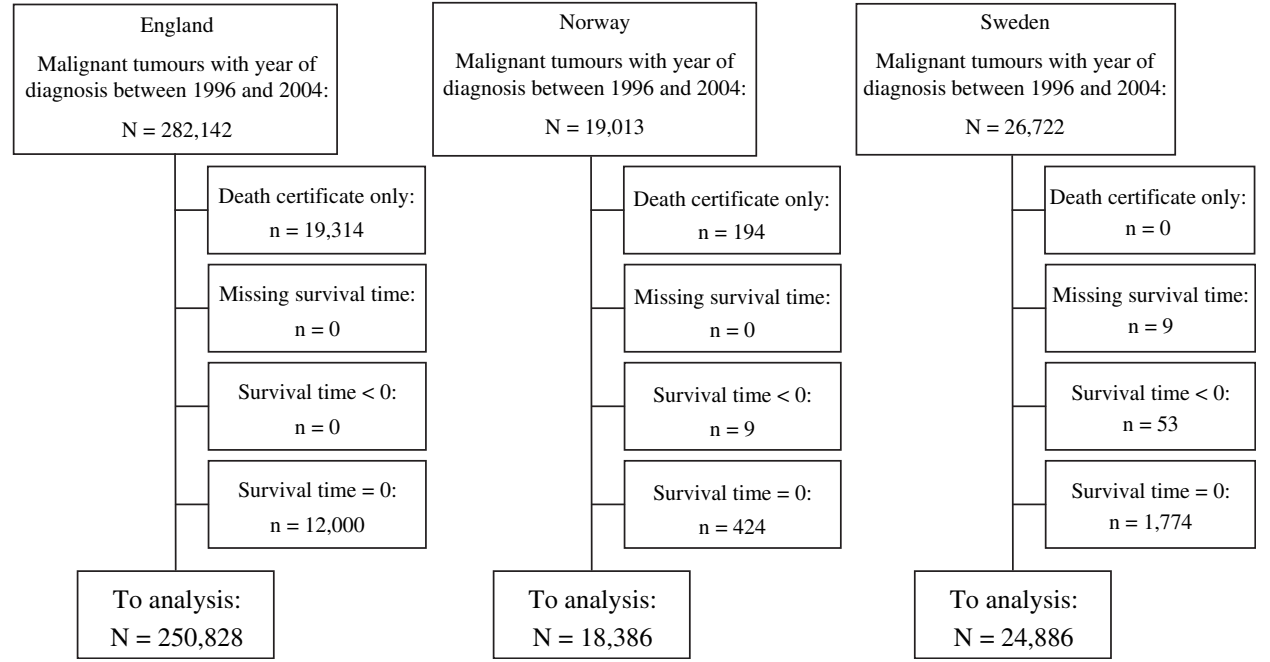

To analysis:

$\mathrm{N}=18,386$ the early follow-up period, diminishing notably after 2 years for men, but less so for women. The largest differences were between England and the other two countries. Differences between Norway and Sweden were smaller and were mainly confined to 3 months follow-up and to older patients (table 3).

\section{Investigation of potential registration artefact phenomenon}

To investigate whether the registration routine in England systematically shortens registered survival times by going backwards from the date of death in search of information in some cases, rather than forwards from the first pathology report, we linked patients with a lung cancer death during the first year of follow-up in England to a hospital inpatient register available from 1997 to 2005, in order to obtain the first known date of a lung cancer diagnosis in an alternative way. This analysis did not materially alter the survival estimates for the English patients.

\section{Effect of digit preference in Norwegian diagnosis dates}

Two additional analyses were performed to assess the impact of the coding of day of diagnosis in the Norwegian cases on estimated survival: keeping the original registered date, and moving the day of diagnosis to day 28 for all patients registered to day 1 . These analyses produced very similar results to the main analysis.

Table 1 Characteristics of included patients with lung cancer, in the period 1996-2004, in England, Norway and Sweden

\begin{tabular}{|c|c|c|c|}
\hline & England & Norway & Sweden \\
\hline & $n=250828$ & $n=18386$ & $n=24886$ \\
\hline \multicolumn{4}{|l|}{ Sex (\%) } \\
\hline Male & $154151(61.5)$ & $11647(63.3)$ & $14391(57.8)$ \\
\hline Female & 96677 (38.5) & $6739(36.7)$ & $10495(42.2)$ \\
\hline \multicolumn{4}{|l|}{ Age at diagnosis } \\
\hline Median & 72 & 70 & 69 \\
\hline First quartile & 64 & 61 & 61 \\
\hline Third quartile & 78 & 76 & 76 \\
\hline \multicolumn{4}{|c|}{ Age group at diagnosis (\%) } \\
\hline $0-49$ & $8824(3.5)$ & $955(5.2)$ & $1182(4.7)$ \\
\hline $50-59$ & $30164(12.0)$ & 3062 (16.7) & $4324(17.4)$ \\
\hline $60-69$ & $65978(26.3)$ & $5114(27.8)$ & $7473(30.0)$ \\
\hline $70-79$ & $98257(39.2)$ & $6699(36.4)$ & 8926 (35.9) \\
\hline $80+$ & $47605(19.0)$ & 2556 (13.9) & $2981(12.0)$ \\
\hline
\end{tabular}

\section{DISCUSSION}

We have found substantial differences in survival between the three countries after a diagnosis of lung cancer, with the worst outcomes observed in England in all age groups. The 5-year relative survival was similar in Norway and Sweden in younger patients, but diverged in patients over 60 . The largest differences occurred early in the follow-up. The absolute differences between countries tended to be greater among women, and women generally had a better prognosis.

The number of deaths was large, and all three countries have good background population data to enable the calculation of relative survival. The methods of registration are well defined for the three registries, and all contain cases verified morphologically, as well as clinically only. The main difference between the countries is that the English data rely more on finding cases via death registration. This can create two biases. ${ }^{14}$ First, the English registration process may miss some patients with a good prognosis. However, in lung cancer, where the great majority of patients have short survival times, this would not severely distort the findings. Secondly, searching retrospectively from the date of death for the date of diagnosis may result in defining the date of a recurrence as the date of primary diagnosis. When we matched the cohort to a hospital inpatient register, the survival estimates were not substantially changed. Again, this potential problem would tend to be minor in a cancer with short survival times. The absence of death certificate-initiated cases in Sweden may, however, account for some of the differences in survival seen between Norway and Sweden. The problem of underreporting in Sweden of elderly patients without histopathological diagnosis and not subjected to treatment is likely to be of similar magnitude in England and Norway. The reassignment of date of diagnosis for Norway was shown to have very little impact on the results.

The Swedish Registry has only recently started to collect stage information, while the Norwegian and English registries do not collect and classify stage information in the same way. Thus, we have no information on stage distribution, which may serve to explain major aspects of the variations observed in this study. The most likely explanation for the observation that the differences between the countries appear early during followup-although this does not necessarily explain all of the observed difference-is that patients in England more frequently present at a late stage. The low proportions of patients offered surgery (as discussed below) make it implausible that the early 
Table 2 5-Year cumulative relative survival, with 95\% Cls in parentheses, in England, Norway and Sweden

\begin{tabular}{|c|c|c|c|c|c|c|}
\hline & \multicolumn{2}{|l|}{ Age 0-49 } & \multicolumn{2}{|l|}{ Age 50-59 } & \multicolumn{2}{|l|}{ Age 60-69 } \\
\hline & Men & Women & Men & Women & Men & Women \\
\hline England & $14.0(12.6$ to 15.4$)$ & $17.8(16.2$ to 19.4$)$ & 9.2 (8.6 to 9.7 ) & $12.2(11.4$ to 13.0$)$ & 7.9 (7.6 to 8.3$)$ & 10.2 (9.7 to 10.8 ) \\
\hline Norway & $17.6(13.5$ to 22.0$)$ & 29.3 (23.5 to 35.3$)$ & $14.7(12.5$ to 17.1$)$ & 21.3 (18.4 to 24.4$)$ & 9.8 (8.4 to 11.3 ) & 13.7 (11.6 to 15.9 ) \\
\hline \multirow{2}{*}{ Sweden } & \multicolumn{2}{|l|}{ Age $70-79$} & \multicolumn{2}{|l|}{ Age $80+$} & \multicolumn{2}{|l|}{ Age standardised } \\
\hline & Men & Women & Men & Women & Men & Women \\
\hline Sweden & 9.9 (8.8 to 11.2$)$ & 13.0 (11.4 to 14.7$)$ & 6.2 (4.3 to 8.7$)$ & 7.1 (4.4 to 10.7$)$ & 11.3 (9.5 to 13.2 ) & $15.9(13.7$ to 18.3$)$ \\
\hline
\end{tabular}

differences are explained in major part by complications of treatment. Late presentation creates a vicious circle: the disease progression is difficult to influence, and the patient's general condition may prohibit antitumour treatment. There is evidence from national audits that the proportion of patients in stage $I$ is of the order of $15 \%$ in England ${ }^{15}$ and $18 \%$ in Sweden. ${ }^{16}$ These estimates are, however, sensitive to completeness of reporting of cases and stage, as well as to intensity of diagnostic investigation, which can lead to stage migration.

Differences in management probably explain part of our results. We have no individual information on treatment in this study, but it is estimated that the proportion of patients with lung cancer offered surgery during the study period was $10.2 \%$ in England, ${ }^{15}$ as opposed to $16.4 \%$ in Norway $^{17}$ and $17 \%$ in Sweden. ${ }^{16}$ The corresponding estimates for the proportion of patients not offered any active antitumour treatment are $48 \%,{ }^{15}$
$40 \%{ }^{18}{ }^{19}$ and $19 \%{ }^{16}$ These figures indicate a generally lower treatment activity in England, but the indications for different treatments are dependent on stage and general health status at presentation. Thus we cannot in this analysis reliably quantify the effects of stage and treatment. The study period lies before the introduction of new treatment modalities such as tyrosine kinase inhibitors. Our results suggest that in-depth studies of diagnostic and therapeutic practices may be highly informative. These studies have to be broad in scope; there is substantial regional variation in results within each country, and a comparison including only a few centres may be misleading. ${ }^{20-22}$

Although the mortality to incidence ratio is close to 1 in all three countries, ${ }^{3}$ lung cancer incidence is higher in England than in Norway and Sweden; world standardised incidence rates for 2000 were 47.6 per 100000 men in the UK, 35.1 in Norway and 21.4 in Sweden. ${ }^{23}$ The corresponding figures for women were
Figure 2 Excess deaths/100 personyears by country, age and follow-up interval during the period 2001-2004.
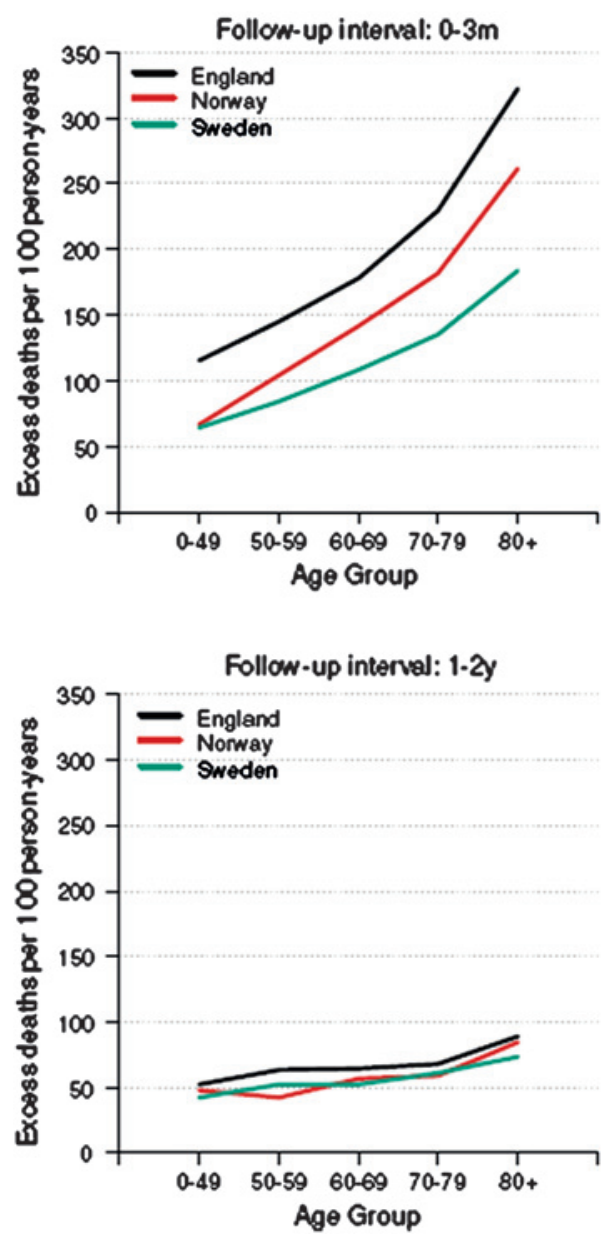
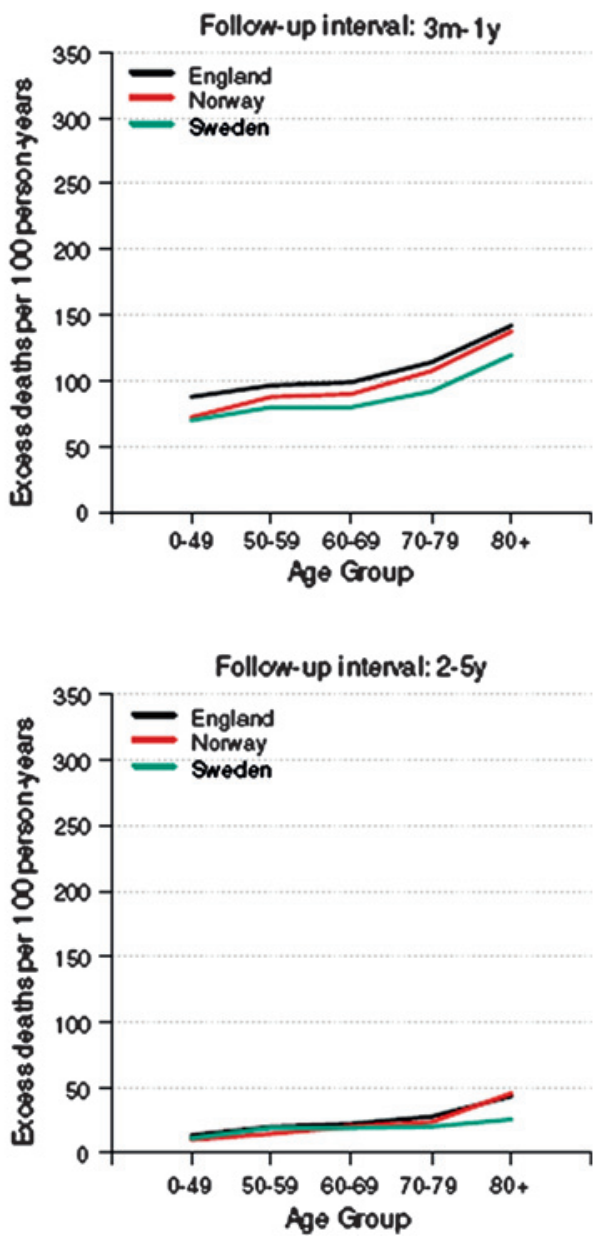
Table 3 Relative excess risk between countries, according to the Poisson model, by sex, age and follow-up interval

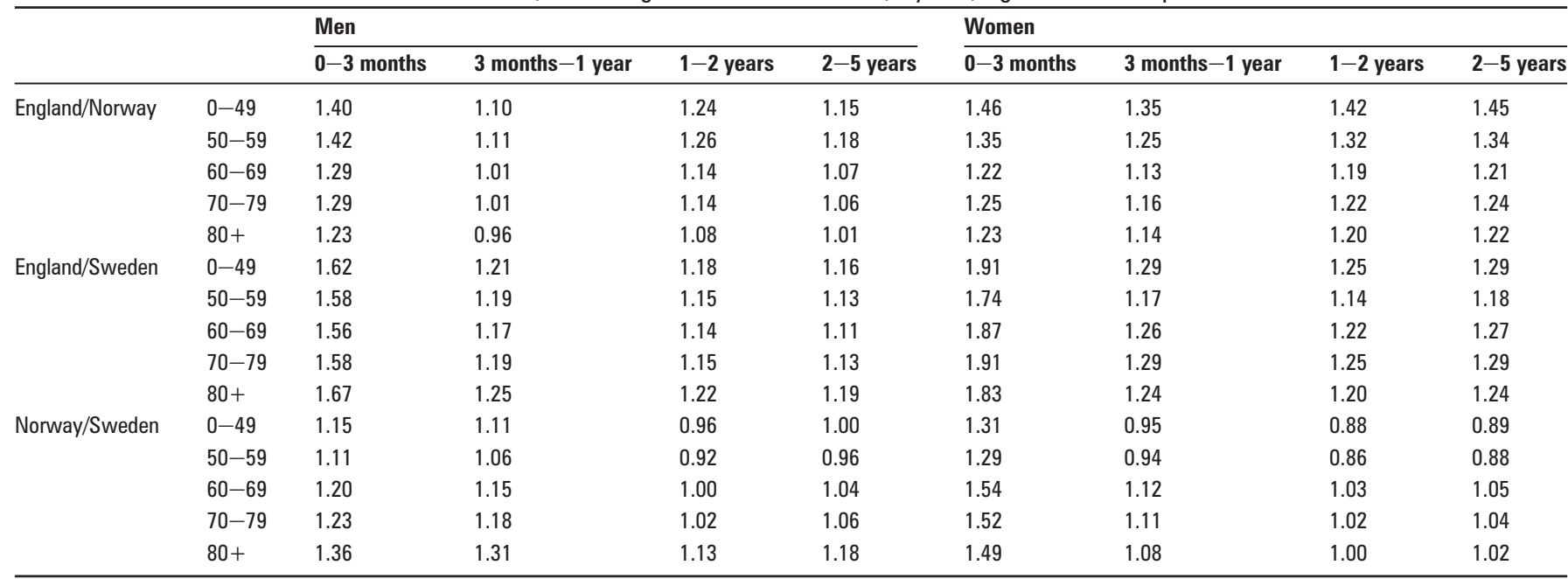

21.8, 16.6 and 12.1. In the UK, incidence and mortality have decreased since the late 1970s from a very high level, and they have stabilised in Norway and Sweden since the late 1980s for men-although for women there has been an increase in incidence and mortality. The incidence and mortality rates reflect different population exposures to tobacco smoking. Prevalence of daily smokers in 2006 was $26.6 \%$ in England and Wales, $24.3 \%$ in Norway and $16.3 \%$ in Sweden. ${ }^{24}$

Patients with lung cancer exhibit a much higher degree of smoking than the background population, from which the comparison mortality estimates are obtained for relative survival calculations. Since smoking is also a strong risk factor for other fatal diseases, a high prevalence of smoking in a cohort of patients with cancer may result in relative survival overestimating the force of cancer mortality in that group. If the proportion of smokers among patients with lung cancer is higher in England than in Norway and Sweden, and the smokers in England have, on average, smoked more heavily and for a longer duration, this could have influenced our results. However, lung cancer in itself has such a severe prognosis that the force of competing mortality from smoking-related disease is likely to be small. Yet, patients heavily exposed to smoking may have sufficiently high levels of co-morbidity as to preclude lung resection, radiotherapy or chemotherapy.

We were not able to compare histopathological types, but there is no marked difference in their distribution in England compared with other European countries, ${ }^{25}$ and any difference in distribution of histopathological type would have to be large in order to explain any substantial part of our results, since the differences in survival by histopathological type are modest. ${ }^{26}$

A better survival among women than men is substantiated in many prognostic studies. ${ }^{27-31}$ Different behaviour of smokinginduced cancer, differing effects of exposure to smoking, different awareness of and response to health messages, and differences in response to treatment by sex may be contributing factors.

Our data have shown that there are clinically relevant differences in lung cancer survival between three countries with similar national expenditure on health and similar healthcare systems. The main threat to the validity of our results is a higher proportion of cancer registrations in England triggered by a death certificate. However, this potential problem is likely to be limited for a disease with generally short survival times, and our own analysis of this potential bias indicated that it did not explain the results to any substantial degree. The finding that the differences are confined mainly to early in the followup is important from several perspectives. First, it points towards the need for detailed studies to determine the reasons for this. Secondly, it suggests that access to healthcare and population awareness are important drivers of these differences. However, we cannot exclude that differences in treatment activity-related or not to co-morbidity-play a role. Thirdly, it indicates that improvements to healthcare systems are likely to result in early improvements in survival in future patient cohorts.

Funding This study was supported by the Department of Health, London, UK.

Competing interests None.

Provenance and peer review Not commissioned; not externally peer reviewed.

\section{REFERENCES}

1. Berrino F, De Angelis R, Sant M, et al; and the EUROCARE Working Group. Survival for eight major cancers and all cancers combined for European adults diagnosed in 1995-99: results of the EUROCARE-4 study. Lancet Oncol 2007:8:773-83.

2. Verdecchia A, Francisci S, Brenner $\mathrm{H}$, et al; and the EUROCARE-4 Working Group. Recent cancer survival in Europe: a 2000-02 period analysis of EUROCARE-4 data. Lancet Oncol 2007:8:784-96.

3. Bray F, Sankila R, Ferlay J, et al. Estimates of cancer incidence and mortality in Europe in 1995. Eur J Cancer 2002;38:99-166.

4. National Cancer Intelligence Network UK. National Cancer Data Repository. http:// www.ukacr.org/ (accessed 30 March 2010).

5. Westlake S. Cancer incidence and mortality in the United Kingdom and constituent countries, 2003-05. Health Stat 0 2008:40:91-7.

6. UK Association of Cancer Registries. Cancer Registration in the UK. http://www.ncin org.uk/ (accessed 30 March 2010).

7. Larsen IK, Småstuen M, Johannesen TB, et al. Data quality at the Cancer Registry of Norway: an overview of comparability, completeness, validity and timeliness. Eur $J$ Cancer 2009;45:1218-31.

8. Barlow L, Westergren K, Holmberg L, et al. The completeness of the Swedish Cancer Register: a sample survey for year 1998. Acta Oncol 2009:48:27-33.

9. Ederer F, Axtell LM, Cutler SJ. The relative survival rate: a statistical methodology. Natl Cancer Inst Monogr 1961;6:101-21.

10. Brenner $\mathbf{H}$, Gefeller $\mathbf{O}$. An alternative approach to monitoring cancer patient survival. Cancer 1996;78:2004-10.

11. Dickman PW, Sloggett A, Hills M, et al. Regression models for relative survival. Stat Med 2004;23:51-64.

12. StataCorp. Stata statistical software: release 9. College Station, Texas: StataCorp LP, 2005.

13. R Development Core Team. $R$ : a language and environment for statistical computing. Vienna, Austria: R Foundation for Statistical Computing, 2007. ISBN 3-900051-07-0, http://www.Rproject.org.

14. Robinson D, Sankila $R$, Hakulinen $T$, et al. Interpreting international comparisons of cancer survival: the effects of incomplete registration and the presence of 
death certificate only cases on survival estimates. Eur J Cancer 2007: 43:909-13

15. UK NHS Information Centre. National Lung Cancer Data Audit (LUCADA). http:// www.ic.nhs.uk/services/national-clinical-audit-support-programme-ncasp/cancer/ lung (accessed 30 March 2010).

16. Regional Oncologic Center in Uppsala/Orebro, Sweden. Lungcancer i Sverige 20022007. http://www.roc.se/lungca.asp (accessed 30 March 2010).

17. Strand TE, Rostad H, Møller B, et al. Survival after resection for primary lung cancer: a population based study of 3211 resected patients. Thorax 2006;61:710-15.

18. Skaug K, Eide GE, Gulsvik A. Prevalence and predictors of symptoms in the terminal stage of lung cancer. Chest 2007;131:389-94.

19. Rolke HB, Bakke PS, Gallefoss F. Delays in the diagnostic pathways for primary pulmonary carcinoma in Southern Norway. Respir Med 2007;101:1251-7.

20. Cartman ML, Hatfield AC, Muers MF, et al. Yorkshire Cancer Management Study Group, Northern and Yorkshire Cancer Registry and Information Service (NYCRIS), UK. Lung cancer: district active treatment rates affect survival. J Epidemiol Community Health 2002:56:424-9.

21. von Plessen C, Strand T-E, Wentzel-Larsen T, et al. Effectiveness of third-generation chemotherapy on the survival of patients with advanced non-small cell lung cancer in Norway: a national study. Thorax 2008:63:866-71.

22. Myrdal G, Lamberg K, Lambe M, et al. Regional differences in treatment and outcome in non-small cell lung cancer: a population-based study (Sweden). Lung Cancer 2009;63:16-22.
23. Curado MP, Edwards B, Shin HR, et al, eds. Cancer incidence in five continents, Vol IX. Lyon: IARC, 2007. IARC Scientific Publications No. 160.

24. WHO. WHO report on the global tobacco epidemic, 2008 The MPOWER package. Geneva: World Health Organization, 2008.

25. Bennett VA, Davies EA, Jack RH, et al. Histological subtype of lung cancer in relation to socio-economic deprivation in South East England. BMC Cancer 2008;8:139-43.

26. Hirsch FR, Spreafico A, Novello $S$, et al. The prognostic and predictive role of histology in advanced non-small cell lung cancer: a literature review. J Thorac Oncol 2008; 3:1468-81

27. Thomas L, Doyle LA, Edelman MJ. Lung cancer in women: emerging differences in epidemiology, biology, and therapy. Chest 2005;128:370-81.

28. Båtevik R, Grong K, Segadal L, et al. The female gender has a positive effect on survival independent of background life expectancy following surgical resection of primary non-small cell lung cancer: a study of absolute and relative survival over 15 years. Lung Cancer 2005;47:173-81.

29. Chian T, Chen $\mathrm{P}, \mathrm{Wu} \mathrm{P}$, et al. Important prognostic factors for the long-term survival of lung cancer subjects in Taiwan. BMC Cancer 2008;8:324.

30. Alexiou C, Onyeaka CVP, Beggs D, et al. Do women live longer following lung resection for carcinoma? Eur J Cardiothorac Surg 2002;21:319-25.

31. Bouchardy C, Fioretta G, De Perrot M, et al. Determinants of long term survival after surgery for cancer of the lung. A population-based study. Cancer 1999:86:2229-37.

\section{Lung alert}

\section{Changes in the DENND1B gene increase the risk of childhood asthma}

Previous studies have shown associations between asthma and single nucleotide polymorphisms (SNPs) on the 17q12 chromosome, but the risk associated with these SNPs is low.

This study used children of European ancestry as a 'discovery set'. Genome-wide association identified several SNPs in the 1q31.3 region associated with childhood onset asthma (typical OR $0.63 ; 95 \%$ CI 0.54 to 0.73 ). The earlier the onset of asthma, the higher the prevalence of risk alleles. These findings were replicated in a different group of European origin. The same locus was associated with asthma in an African-American cohort, but in these children the alleles associated with asthma were the opposite alleles to those in the European subjects. This may be related to modifying effects of other genes, or differences in linkage disequilibrium.

The SNPs with the strongest effect were within the intron of the DENND1B gene. This gene is expressed in dendritic cells and activated T cells and modulates the type 1/type 2 helper T cell response.

It seems likely that changes in the DENND1B gene alter the risk of childhood asthma. However, the risk attributable to this gene is low-the protective allele frequency of the strongest associated SNP was $15.2 \%$ in patients with asthma and $22.2 \%$ in controls. Single genes may confer an increased risk of certain asthma phenotypes, but their effect is modified by multiple other genetic and environmental factors.

Sleiman PMA, Flory J, Imielinski M, et al. Variants of DENND1B associated with asthma in children. $N$ Engl J Med 2010;362:36-44.

\section{S Haney}

Correspondence to S Haney, Wansbeck Hospital, Northumbria, UK; sas haney@yahoo.co.uk

Thorax 2010;65:441. doi:10.1136/thx.2010.136283 